\title{
New force and new heat
}

\author{
Yuriy Alexeevich Baurov ${ }^{1,2}$, Lorenzo Albanese ${ }^{3}$, Francesco Meneguzzo ${ }^{3,}$ * \\ ${ }^{1}$ Closed Joint Stock Company Research Institute of Cosmic Physics, 141070, Moscow Region, Pionerskaya, 4, Korolyov, Russia \\ ${ }^{2}$ Hotwater Srl, Via Gioberti, 15, I-56024 San Miniato (PI), Italy \\ ${ }^{3}$ National Research Council, Institute of Biometeorology, 8, Via G, Caproni, I-50145 Firenze, Italy
}

Email address:

baurov@mail.ru (Y. A. Baurov), 1.albanese@ibimet.cnr.it (L. Albanese), f.meneguzzo@ibimet.cnr.it (F. Meneguzzo)

\section{To cite this article:}

Yuriy Alexeevich Baurov, Lorenzo Albanese, Francesco Meneguzzo. New Force and New Heat. American Journal of Astronomy and Astrophysics. Special Issue: Global Anisotropy, Theory of Byuon, New Force, New Power System, Propulsion, Space Flights.

Vol. 2, No. 6-1, 2014, pp. 47-53. doi: 10.11648/j.ajaa.s.2014020601.17

\begin{abstract}
Total heat generation in a vertically-shaped closed hydraulic circuit of specific section, supplied with a special device (SD) used to lock the circulating liquid medium, i.e. regular water, is analyzed in order to detect possible excess heat with regards to input electric or mechanical power. Long experimental runs were carried out in order to extract transient continuous equilibrium periods, i.e. constant water temperature, distinguished by the imposed recovery pressure and the SD model, while equilibrium series were obtained after connecting the main circuit to a heat exchanger with adjustable dissipation power. The generated heat was measured after observing the flow to the heat exchanger and the temperature difference between the supply and return lines. The heat dissipation from the main circuit was measured as a function of the difference between the circuit and environmental temperatures, as well as a calibration test without any SD was carried out in order to estimate the pump's motor efficiency on the basis of classical energy conservation. The results show a sharp dependence of excess heat generation from height of installation and statistically significant dependence of excess heat generation upon both water temperature and hydraulic pressure. This evidence agrees with predictions from the theory of byuons about the occurrence of a new force of nature acting upon quickly rising substance in the Earth's gravitational field, the work performed by such force increasing with the vertical distance traveled by the substance. The results of experiments with heat installation practical show a decision of problem of A. Einstein, i.e. the creation of Joint Theory of Fields.
\end{abstract}

Keywords: New Force, Theory of Byuon, New Heat

\section{Introduction}

In papers [1-3] the results of experimental investigations are described, in which, with the aid of high current magnets, torsion and piezoresonance balances, an interaction of the objects of nature, different from the known ones - the strong, weak, electromagnetic and gravitational - was first detected.

The value of the detected force was around $(0.01-0.08) \mathrm{g}$ in a magnetic field of magnitude $\approx(10-13) \mathrm{T}$ with aperture of the coils in the range (40-53) mm and loads' weight (26-30) g.

Further experimental studies of the new proposed interaction by means of gravimeters with magnets arranged nearby [4-6], as well as studies of changes in the rate of $\beta$ decay of radioactive elements [5-10], confirmed the results obtained in [1-3].

The key feature of the new interaction is its anisotropy, shown in a wide range of sizes, from the characteristic dimensions of $10^{-17} \mathrm{~cm}$ of the weak interactions [5-10] to the size of our Galaxy $\left(10^{22} \mathrm{~cm}\right)[11,12]$. However, the new interaction shows as well isotropic properties [5,6]. It is noticeable that in [13], on the basis of a new interaction, an explanation is given about the origin of dark energy as a cause of receding galaxies with acceleration.

The physical nature of the new force, in accordance with the Byuon theory (BT) (non-gauge theory of the formation of physical space and the world of elementary particles on the basis of unobservable objects named "byuons") [5,6], appears to affect the formation of masses of elementary particles by means of potentials of physical fields because the fraction of the mass of elementary particles associated with the formation of their internal physical space in the frame work of the Byuon theory is proportional to the modulus of some summary potential $A_{\Sigma}$, which size can't be greater than the modulus of the cosmological vector potential $A_{g}$ which is the new fundamental constant, introduced in [5,6] (module of $\mathrm{A}_{\mathrm{g}}$ is equal to $\left.1.9 \cdot 10^{11} \mathrm{Gs} \cdot \mathrm{cm}\right)$. Hence the new force will push out any material body from the region of the decreased modulus of $A_{\Sigma}$, because a defect of energy $E=m \cdot c^{2}$ will arise 
and the corresponding force will point to the region with undisturbed value of $\mathrm{A}_{\Sigma}$.

The most accurate results to determine the anisotropic properties of the new force were found in studies using plasma devices $[6,14,15]$, which showed that the new force rejects substance out of the region of weakened summary potential $\mathrm{A}_{\Sigma}$ along the generatrix of a cone with an opening of $100^{\circ}-110^{\circ}$ around the vector $A_{g}$ that sets the global spatial anisotropy of physical space and has the coordinates $\alpha \approx 293^{\circ} \pm 10^{\circ}$ (right ascension) and $\delta \approx 36^{\circ} \pm 10^{\circ}$ (declination) in the second equatorial coordinate system.

The finding of the anisotropic properties of physical space is supported by many astrophysical observations: the anisotropy of the motion of the pulsars in the plane of the sky $[6,11,12]$, the anisotropy of the distribution of solar flares on the Sun's surface [5,6], the anisotropy of the distribution of earthquakes on the Earth $[6,16]$ with respect to the stars (the most powerful earthquakes in the northern hemisphere occur when the vector potential of the Earth magnetic field during the planet's rotation is directed antiparallel to the vector $A_{g}$ ).

Results of experimental investigation of plasma vacuum bubbles resulting from hydrodynamic cavitation in a water filled closed circuit showed some excess water heating becoming significant during abrupt changes of the polarity of the southward component of the interplanetary magnetic field (IMF) frozen in the solar wind, which is compatible with the action of a new force of nature predicted by the non-gauge cosmological physical theory of byuons [17]. These results were achieved at temperatures below $40^{\circ} \mathrm{C}$, as well as showed that the excess heating increased with the hydraulic pressure at least up to a certain value, in turn agreeing with the hypothesis of the activation of the new force after interaction of the IMF with the plasma vacuum bubbles typical of violent $\mathrm{HC}$ occurring at low water temperatures and high enough pressures [18].

The present article is devoted to the heating of water at high temperatures (up to nearly $100^{\circ} \mathrm{C}$ ) using solution to the Joint Theory of Fields (JTF) based on the BT.

\section{Theoretical Insights}

\subsection{The Global Anisotropy of the Physical Space and the Byuon Theory}

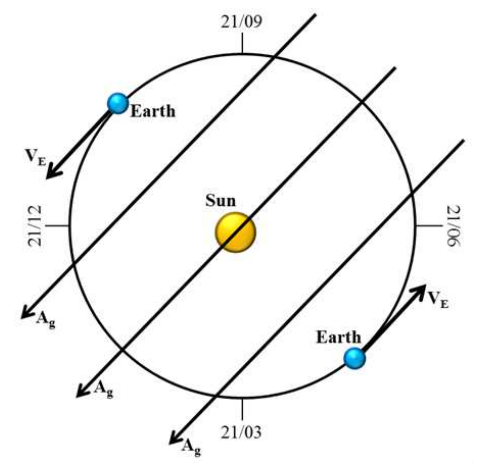

Fig. 1. Projection of the cosmological vector potential $A_{g}$ onto a simplified Earth's orbital plane; $V_{E}$ is the Earth's instantaneous velocity vector.
In [19] the vector $A_{g}$ and therefore the global anisotropy of the physical space was determined, having the following astronomical coordinates in the second equatorial system: $\alpha \approx 300^{\circ} \pm 10^{\circ}, \delta \approx 36^{\circ} \pm 10^{\circ}$, where $\alpha$ is the right ascension and $\delta$ is the declination, as the most exact results so far.

Fig. 1 shows the direction of the cosmological vector potential $A_{g}$ projected onto the Earth's orbital plane, representing the direction of the global anisotropy of the physical space.

The analysis of a long run of experiments has shown that the new force has a nonlinear and nonlocal character and can be represented as a complex series in terms of changes of the summary potential $A_{\Sigma}$ surface [5,6]. The first term of the series is the following:

$$
F=2 N m_{v} c^{2} \lambda_{1}^{2} \Delta A_{\Sigma}\left[\Delta\left(\Delta A_{\Sigma}\right) / \Delta x\right]
$$

where $N$ is the number of stable particles (electrons, protons, and neutrons) in the test body, $\Delta A_{\Sigma}$ is the difference in changes of the summary potential $A_{\Sigma}$ at the location points of a test body and sensor element, $\Delta\left(\Delta A_{\Sigma}\right) / \Delta x$ is the gradient in space of the difference potentials $\Delta A_{\Sigma} ; x$ is the length of an arc of a circle for experiments with solenoids, therefore a space coordinate; $2 m_{v} c^{2}=33 \mathrm{eV} ; \lambda_{l}=10^{-6}(\mathrm{~T} \cdot \mathrm{m})^{-1}$ is the first coefficient of the series.

The fundamental research of the global anisotropy of physical space along with the basics of the byuon theory based upon such anisotropy is summarized in Baurov [19] which includes all the relevant bibliography.

\subsection{Processes Leading to Excess Heat Generation}

The bulk heat generation in a closed hydraulic circuit including a centrifugal pump powered by electric energy originates from the conversion of the mechanical energy input supplied by the pump's rotor to the circulating liquid, occurring through several different friction processes. Any excess heat, i.e., any alleged violation of the classical energy conservation, can be detected only after subtracting the trivial contribution provided by the pump. It should be noted that the energy dissipation caused by the pump's motor limited efficiency should as well be excluded from the balance, even if it can contribute to reduce the circuit to environment temperature gradient, therefore re-entering the balance through the circuit heat loss component.

Two main mechanisms based upon the theory of byuons are proposed to explain the generation of excess heat in a vertically shaped closed hydraulic circuit equipped with an SD.

The first mechanism occurs through the collapse of plasma vacuum cavitation bubbles and is fully explained in a previous work [17]. As recalled in Section 1, plasma vacuum bubbles practically disappears when water is warmer than $40^{\circ} \mathrm{C}$, while at lower temperatures the extent of the additional heating process is strongly dependent upon transient and unpredictable events such as IMF polarity inversion; as a result, while preserving a high scientific interest, the process is hard to detect and of little practical use. 
The second and main additional heating process associated with the new force of nature is due to the decrease of the summary potential $A_{\Sigma}$ due to the gravitational potential which, being negative, increases with height from the local Earth's surface, therefore as per Eq. (1) a particle rising from the surface with high enough speed is affected by an upward force towards higher values of the summary potential. Such process is analogous to one proposed to explain some of the accumulation of energy in violent tornadoes [20]. Additional heating is generated due to friction slowing the vertically accelerated particles.

Under this hypothesis, the change in summary potential $\Delta A_{\Sigma}$ can be computed from the already mentioned energy relation (Eq. (3.1.) in [21]), i.e.:

$$
e_{0}\left|\Delta A_{2}\right| \sin \gamma=m_{p} g h
$$

where $e_{0}$ is the proton's electric charge, $m_{p}$ is its mass, $g$ is gravitational acceleration, $h$ is the height travelled by the rising water, $\sin \gamma \approx 10^{-15}$ is a parameter estimated in previous works $[5,6]$, characterizing the anisotropic properties of the physical space. Eq. (2) represents a simple consequence of BT in the direction to a JTF, yet the fundamental solution of the JTF problem by means of the BT is given on the base of fundamental physical scale of changes in our world $\left(10^{-17} \mathrm{~cm}\right.$, $10^{-13} \mathrm{~cm}$ ) using potentials of physical fields $[5,6]$.

Assuming $100 \mathrm{~kg}$ of water, we have: $N \approx 0.38 \cdot 10^{29}$, then from Eq. (2): $\left|\Delta A_{2}\right| \approx 5 \cdot 10^{6} \mathrm{G} \cdot \mathrm{cm}$ and from Eq. (1) the force would be around $2.5 \cdot 10^{9} \mathrm{~N}$ that, along a vertical travel of $4 \mathrm{~m}$ would produce a work around $10^{10} \mathrm{~J}$. Bringing the same amount of water from $0^{\circ} \mathrm{C}$ to $100^{\circ} \mathrm{C}$ requires a heat equal to $2.5 \cdot 10^{8} \mathrm{~J}$, therefore the proposed mechanism could supply all the needed energy. Even if using the linear approximation for the new force as in Eq. (1) is known to lead to an overestimation by about one order of magnitude [5,6], the new force would still able to bring $100 \mathrm{~kg}$ of water from the freezing to boiling point in about $1 \mathrm{~m}$ of rising if applied to all water particles.

Practically, in a closed hydraulic circuit, only a fraction of the circulating water is rising at any given moment; moreover and more subtly, the new force can act upon a substance's particle only during a time depending on the extinction rate of its information object (IO) and anyway less than $1 \mathrm{sec}$, with an intensity decreasing in time, such extinction rate moreover changing in time with a major seasonal shift observed in early February along with other changes scattered during the year and even at the daily and sub-daily scale $[22,23]$. The latter limitation reflects into smaller than expected work to be performed by the new force over most of the water particles in a closed circuit.

A third and final mechanism leading to excess heating is predicted, activating when the direction of the cosmological vector potential $\mathrm{A}_{\mathrm{g}}$ lies around the horizontal plane tangent to the local Earth's surface: this process results in the generation of further rotation around the vertical axis of any particle while rising in the circuit, at all analogous to the main energy accumulation process suggested for natural tornadoes [20]. Additional heating is generated due to friction slowing the induced rotation.

Few important directions to optimize heat installations based upon BT are derived from early experiments: the main pipe diameter must be around $10 \mathrm{~cm}$ (scale of IO of elementary particles); the height of the installation must be greater than for older installations (i.e., more than $2.6 \mathrm{~m}$ ); finally, electric consumption can be minimized by means of an SD.

\section{Experimental Set-Up and Method of Investigation}

A closed hydraulic circuit powered by a centrifugal pump with nominal mechanical power equal to $4 \mathrm{~kW}$, vertical extent over the pump's exit around $4 \mathrm{~m}$ and main pipe diameter about $100 \mathrm{~mm}$, was connected to an heat exchanger by means of a supply and return line, in turn equipped with a calibrated flow meter having resolution 0.1 liters $/ \mathrm{min}$.

Regular water was supplied to the circuit, $75 \pm 1$ liters needed to fill the main circuit plus about 10 liters filling the secondary circuit to the heat exchanger; the circuit was equipped with several calibrated digital temperature and pressure gauges with precision $0.01^{\circ} \mathrm{C}$ and 0.1 bar, respectively; thermometers were in direct contact with water, 8 sensors along the main circuit and 2 gauges along the supply and return lines connecting the main circuit with the heat exchanger, each one about $3 \mathrm{~cm}$ far from the main circuit. The heat exchanger consisted of a commercial hot air fan with heating power $3 \mathrm{~kW}$ to $8 \mathrm{~kW}$. A special device (SD) in the form of a nozzle was installed about $0.8 \mathrm{~m}$ above the pump's exit, while the recovery pressure upstream the SD was regulated by means of a 50-liters expansion tank.

The electricity consumed by the centrifugal pump was recorded by a digital Watt-meter with resolution $0.01 \mathrm{kWh}$.

Fig. 2(a,b) shows a scheme and a picture of the experimental installation.

The whole installation was placed in a closed building; two further thermometers were fixed at about $1 \mathrm{~m}$ and $3 \mathrm{~m}$ above the floor at an average distance of $1.5 \mathrm{~m}$ from the main circuit, in order to measure the environmental temperature.

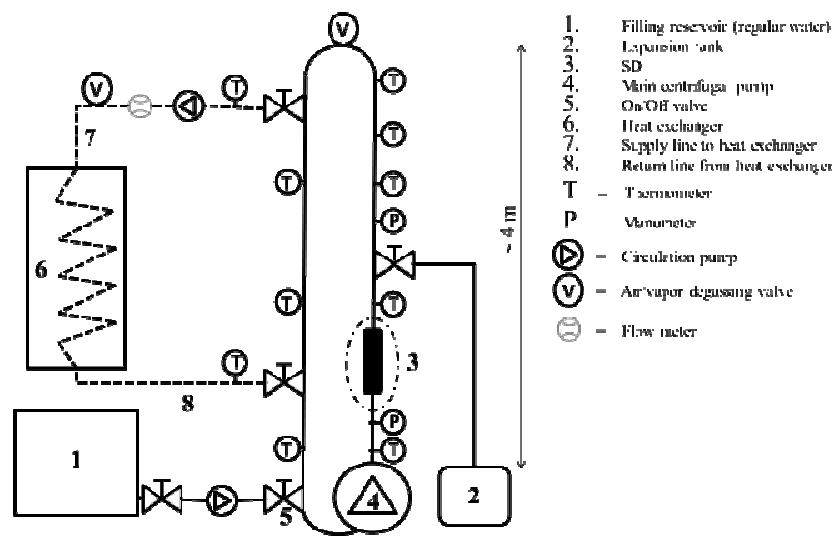

(a) 


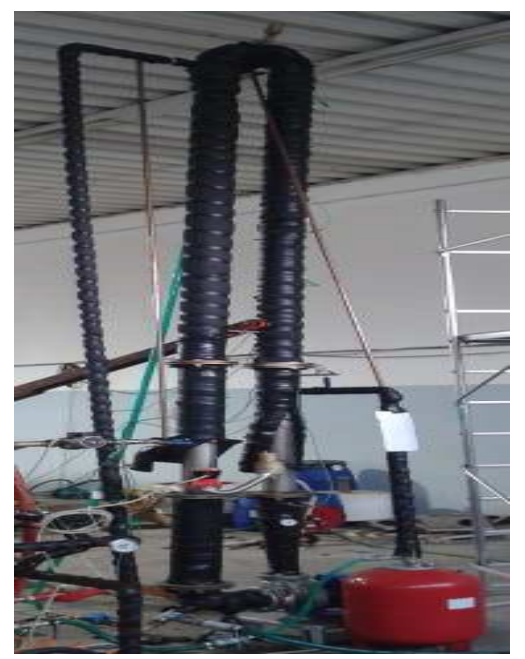

(b)

Fig. 2. Experimental installation: schematic view (a) and picture of the main circuit (b).

The heat loss from the main circuit, reduced by its thermal insulation, was assessed observing the simultaneous evolution of water and environmental temperature every minute during about 900 minutes; the water and environmental temperatures were computed as the arithmetic averages over the 8 sensors along the main circuit, and the 2 external sensors, respectively; the heat power loss was computed assuming a water mass of $75 \mathrm{~kg}$ (i.e., neglecting the mass of metallic structures) and its heat capacity approximated to $4186 \mathrm{~J} /(\mathrm{kg} \cdot \mathrm{K})$.

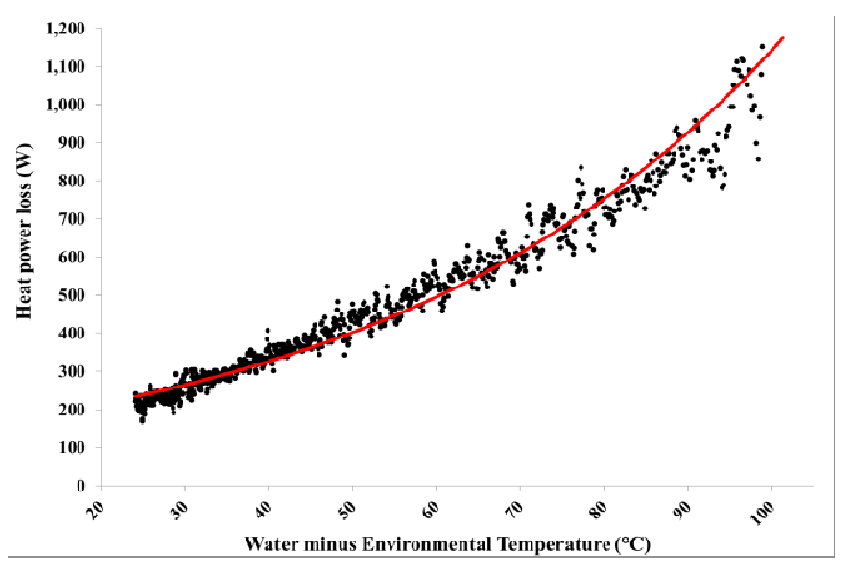

Fig. 3. Projection of the cosmological vector potential $A_{g}$ onto a simplified Earth's orbital plane; $V_{E}$ is the Earth's instantaneous velocity vector.

Fig. 3 shows the heat loss power from the main circuit as a function of the difference between its water temperature and the environmental temperature.

Despite the apparent linearity up to more than $70^{\circ} \mathrm{C}$ temperature difference, the interpolation curve in Fig. 3 is best fitted by an exponential function:

$$
\mathrm{dQ}_{\text {loss }} / \mathrm{dt}=141.17 \cdot \mathrm{e}^{(0.0209 \cdot \Delta \mathrm{T})}
$$

where $d Q_{\text {loss }} / d t$ is the heat power loss $(\mathrm{W}), \Delta T$ is the water minus environment temperature difference $\left({ }^{\circ} \mathrm{C}\right)$.
All sensors recorded data every minute, therefore the time interval dt is always $60 \mathrm{~s}$.

A "blank" test was carried out without any SD in order to estimate the pump's motor efficiency, which is needed in order to compute the correct energy balance for any other experiments, as explained at the beginning of Section 2.2. Such parameter was estimated after forcing the heat generation in this test to be equal to the mechanical input power, which of course is regulated by the motor's efficiency.

The gross heat generation was computed as follows:

$$
d Q / d t=4186 \cdot(d V / d t) \cdot \Delta T+d Q_{l o s s} / d t
$$

where $d Q / d t$ is the heat power generation $(\mathrm{W}), d V / d t$ is the water flow (1/s), $\Delta T$ is the temperature difference between the supply and return lines connecting the main circuit to the heat exchanger $\left({ }^{\circ} \mathrm{C}\right), d Q_{\text {loss }} / d t$ is the heat power loss from Eq. (3).

The pump's motor efficiency was estimated at the level of $93 \%$ by equaling the result from Eq. (4) to the power consumed by the pump multiplied by the motor's efficiency during an equilibrium period covering $84 \mathrm{~min}$, when both the standard deviation and the maximum amplitude of the water temperature range didn't exceed $0.3^{\circ} \mathrm{C}$.

Two models of SD were used for all the other experiments, namely an orifice plate and a Venturi tube, both with total opening about $6 \%$ of the main pipe's section.

The whole installation, including the main and secondary pipes, the SD, the pump and any detail were made of foodquality non-magnetic stainless steel (AISI 304).

\section{Results and Discussion}

Besides the blank test, a total of 20 experiments were performed with an SD included in the hydraulic circuit represented in Fig. 2.

Two measures of performance were computed: the Coefficient of Performance (C.O.P.), as the ratio between the heat generation computed according to Eq. (4) to the consumed electricity measured by the Watt-meter, and the mechanical C.O.P. (hereinafter C.O.P.mech) as the ratio between the heat generation computed according to Eq. (4) and the consumed electricity multiplied by the pump's rotor efficiency, therefore independent of the motor's efficiency. Any generation of excess heat should be revealed by a significant departure of C.O.P.mech from unity.

The list of such experiments along with their main respective features is available in Table 1.

In addition to water temperature, pressure was exactly stable during any experiment listed in Table 1; standard deviations of C.O.P. and C.O.P.mech were around $4 \%$ of the respective averages with few peaks as great as $11 \%$. The power consumed by the pump was observed to change in the range about $4500 \mathrm{~W}$ to $5000 \mathrm{~W}$ in different experiments, while being stable in any single test (standard deviation not greater than $20 \mathrm{~W}$ ).

Fig. 4 shows the values of C.O.P.mech along with the respective uncertainties for all experiments listed in Table 1. 


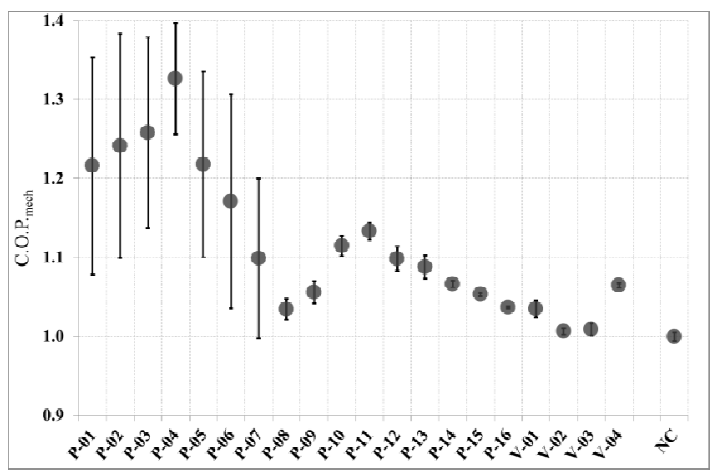

It arises that a positive excess heat is significant in most experiments, with a peak of C.O.P.mech at $1.33 \pm 0.07(\mathrm{P}-04)$, while the C.O.P. exceeds unity in about one third of the experiments (i.e., the generated heat power exceeds the consumed electric power).

Moreover, a close look at the data in Table 1 shows that C.O.P.mech increases with water temperature and decreases with pressure, at least until temperature is lower than $90^{\circ} \mathrm{C}$ and pressure is not too low.

Fig. 4. Series of values of the mechanical Coefficient of Performance for all experiments listed in Table 1.

Table 1. Main features of the performed experiments

\begin{tabular}{|c|c|c|c|c|c|c|c|}
\hline Test ID & $\begin{array}{l}\text { Date and } \\
\text { Time interval (GMT) }\end{array}$ & SD & $\begin{array}{l}\text { Average } \\
\text { Temperature }\left({ }^{\circ} \mathbf{C}\right)\end{array}$ & $\begin{array}{l}\text { Average Pressure } \\
\text { (bar) }\end{array}$ & $\begin{array}{l}A_{g} \quad \text { vector average } \\
\text { altitude (degrees) }\end{array}$ & C.O.P. & $\begin{array}{l}\text { C.O.P.mec } \\
\text { h }\end{array}$ \\
\hline P-01 & $\begin{array}{l}\text { February } 4^{\text {th }}, 2014 \\
09^{\text {h }} 01^{\prime}-11^{\text {h }} 32^{\prime}\end{array}$ & Orifice plate & 83.74 & 1.60 & 82.6 & 1.13 & 1.22 \\
\hline P-02 & $\begin{array}{l}\text { February } 4^{\text {th }}, 2014 \\
11^{\mathrm{h}} 37^{\prime}-13^{\mathrm{h}} 23^{\prime}\end{array}$ & Orifice plate & 83.94 & 4.00 & 63.7 & 1.15 & 1.24 \\
\hline P-03 & $\begin{array}{l}\text { February } 4^{\text {th }}, 2014 \\
13^{\text {h }} 28^{\prime}-14^{\text {h }} 45^{\prime}\end{array}$ & Orifice plate & 83.99 & 0.55 & 46.0 & 1.17 & 1.26 \\
\hline P-04 & $\begin{array}{l}\text { February } 4^{\text {th }}, 2014 \\
15^{\text {h }} 14^{\prime}-17^{\text {h }} 00^{\prime}\end{array}$ & Orifice plate & 83.46 & 2.30 & 25.3 & 1.23 & 1.33 \\
\hline P-05 & $\begin{array}{l}\text { February } 5^{\text {th }}, 2014 \\
15^{\mathrm{h}} 02^{\prime}-15^{\mathrm{h}} 59^{\prime}\end{array}$ & Orifice plate & 98.06 & 2.40 & 30.8 & 1.13 & 1.22 \\
\hline P-06 & $\begin{array}{l}\text { February } 6^{\text {th }}, 2014 \\
08^{\mathrm{h}} 46^{\prime}-11^{\mathrm{h}} 40^{\prime}\end{array}$ & Orifice plate & 84.74 & 3.00 & 80.3 & 1.09 & 1.17 \\
\hline P-07 & $\begin{array}{l}\text { February } 7^{\text {th }}, 2014 \\
07^{\mathrm{h}} 32^{\prime}-08^{\mathrm{h}} 18^{\prime}\end{array}$ & Orifice plate & 81.55 & 3.00 & 62.9 & 1.02 & 1.10 \\
\hline P-08 & $\begin{array}{l}\text { February } 18^{\text {th }}, 2014 \\
02^{\mathrm{h}} 39^{\prime}-05^{\mathrm{h}} 12^{\prime}\end{array}$ & Orifice plate & 57.20 & 2.60 & 29.6 & 0.96 & 1.03 \\
\hline P-09 & $\begin{array}{l}\text { February } 18^{\text {th }}, 2014 \\
05^{\text {h }} 49^{\prime}-06^{\text {h }} 54^{\prime}\end{array}$ & Orifice plate & 56.90 & 2.60 & 54.4 & 0.98 & 1.06 \\
\hline P-10 & $\begin{array}{l}\text { February } 18^{\text {th }}, 2014 \\
14^{\text {h }} 39^{\prime}-16^{\text {h }} 32^{\prime}\end{array}$ & Orifice plate & 61.39 & 2.00 & 20.7 & 1.04 & 1.11 \\
\hline P-11 & $\begin{array}{l}\text { February } 18^{\text {th }}, 2014 \\
16^{\text {h }} 56^{\prime}-17^{\text {h }} 26^{\prime}\end{array}$ & Orifice plate & 61.44 & 1.50 & 7.6 & 1.05 & 1.13 \\
\hline P-12 & $\begin{array}{l}\text { February } 18^{\text {th }}, 2014 \\
20^{\text {h }} 34^{\prime}-22^{\text {h }} 01^{\prime}\end{array}$ & Orifice plate & 60.30 & 3.20 & -11.0 & 1.02 & 1.10 \\
\hline P-13 & $\begin{array}{l}\text { February } 19^{\text {th }}, 2014 \\
02^{\mathrm{h}} 08^{\prime}-04^{\mathrm{h}} 00^{\prime}\end{array}$ & Orifice plate & 59.43 & 3.20 & 21.2 & 1.01 & 1.09 \\
\hline P-14 & $\begin{array}{l}\text { April } 14^{\text {th }}, 2014 \\
15^{\text {h }} 13^{\prime}-15^{\text {h }} 32^{\prime}\end{array}$ & Orifice plate & 58.92 & 3.80 & -4.4 & 0.99 & 1.07 \\
\hline P-15 & $\begin{array}{l}\text { April 14 } 4^{\text {th }}, 2014 \\
17^{\mathrm{h}} 49^{\prime}-18^{\mathrm{h}} 33^{\prime}\end{array}$ & Orifice plate & 57.31 & 2.60 & -10.8 & 0.98 & 1.05 \\
\hline P-16 & $\begin{array}{l}\text { April } 14^{\text {th }}, 2014 \\
21^{\mathrm{h}} 01^{\prime}-21^{\mathrm{h}} 33^{\prime}\end{array}$ & Orifice plate & 56.69 & 4.00 & 2.4 & 0.96 & 1.04 \\
\hline $\mathrm{NC}$ & $\begin{array}{l}\text { June } 3^{\text {rd }}-4^{\text {th }}, 2014 \\
23^{\mathrm{h}} 25^{\prime}-00^{\mathrm{h}} 48^{\prime}\end{array}$ & $\begin{array}{l}\text { None } \\
\text { ("blank") }\end{array}$ & 58.53 & 3.00 & 63.0 & 0.95 & 1.00 \\
\hline V-01 & $\begin{array}{l}\text { June } 5^{\text {th }}, 2014 \\
16^{\mathrm{h}} 44^{\prime}-17^{\mathrm{h}} 07^{\prime}\end{array}$ & Venturi tube & 65.61 & 5.10 & -3.5 & 0.96 & 1.04 \\
\hline V-02 & $\begin{array}{l}\text { June } 5^{\text {th }}, 2014 \\
21^{\mathrm{h}} 34^{\prime}-21^{\mathrm{h}} 47^{\prime}\end{array}$ & Venturi tube & 44.34 & 4.90 & 37.3 & 0.94 & 1.01 \\
\hline V-03 & $\begin{array}{l}\text { June } 5^{\text {th }}, 2014 \\
22^{\mathrm{h}} 15^{\prime}-23^{\mathrm{h}} 29^{\prime}\end{array}$ & Venturi tube & 44.05 & 4.90 & 49.8 & 0.94 & 1.01 \\
\hline V-04 & $\begin{array}{l}\text { October } 14^{\text {th }}, 2014 \\
14^{\text {h }} 02^{\prime}-14^{\text {h }} 38^{\prime}\end{array}$ & Venturi tube & 62.49 & 2.50 & 50.7 & 0.99 & 1.06 \\
\hline
\end{tabular}

Despite the apparent non-linearity of the dependence of the excess heat generation upon temperature and pressure, a simple linear multiregression of C.O.P.mech against those quantities explains as much as $76 \%$ of variance, better than against any other pair of quantities. The expression is the following: 


$$
\text { C.O.P. } \text { mech }=(0.86 \pm 0.08)+(0.005 \pm 0.0008) \cdot \mathrm{T}+(0.017 \pm 0.010) \cdot \mathrm{P}
$$

where $T$ is the water temperature and $P$ is the hydraulic recovery pressure. The uncertainty affecting C.O.P.mech is about $4 \%$ of its average value over the all the experiments.

Fig. 5 shows the comparison between the observed values of C.O.P. mech $_{\text {and }}$ its reconstruction on the basis of Eq. (5); both orifice plate and Venturi tube experimental data smoothly fit along an approximate straight line. Nevertheless, a striking positive outlier, i.e. with observed value much higher than the computed one, as well as absolute maximum across the whole set of experiments (experiment P-04), and at least two minor ones, positive and negative, are apparent.

In order to try an interpretation of such outliers, the series of the deviations of the observed values of C.O.P. mech $_{\text {from }}$ their computed counterparts are shown along with different physical quantities in Fig. 6(a-c).

Fig. 6c shows that the outlier corresponding to experiment P-04 occurred when the altitude of the cosmological vector $\mathrm{A}_{\mathrm{g}}$ was the lowest among all experiments carried out at temperatures higher than $75^{\circ} \mathrm{C}$, including two other experiments having lower pressure and one having similar pressure (Fig. 6b).

The latter argument could support the hypothesis previously advanced about the energy accumulation mechanism of tornadoes [20].

Furthermore, it should be observed the abrupt transition to underestimation of C.O.P.mech (Fig. 6) and lower energy performances (Fig. 4) from P-04 to P-05 and P-06, i.e. from February $4^{\text {th }}$ to February $5^{\text {th }}$ and February $6^{\text {th }}, 2014$, which can hardly be explained on the basis of the ordinary physical quantities such as temperature and pressure (Fig. 6), thereby pointing to a role of the IO extinction rate as already observed in previous experiments with a space thruster, as mentioned in Section 2.2.

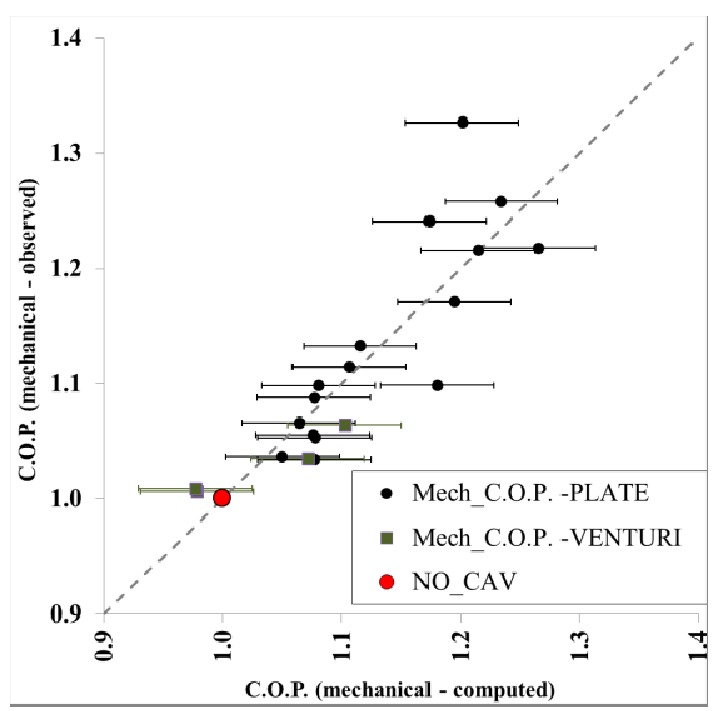

Fig. 5. Comparison between observed and computed mechanical C.O.P. for the whole set of experiments; experiments with the orifice plate and Venturi tube as SD, as well as the blank test with no SD ("NO_CAV"), are represented with different colors.

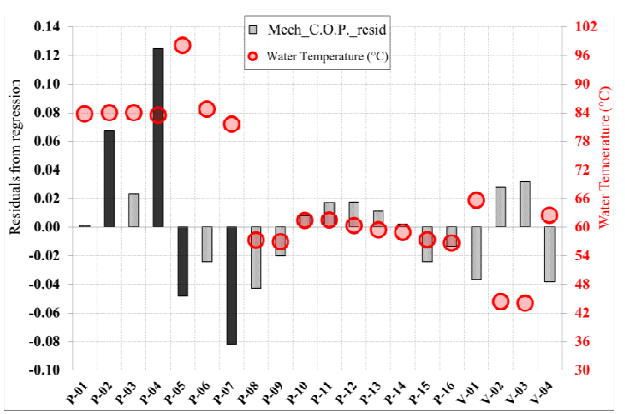

(a)

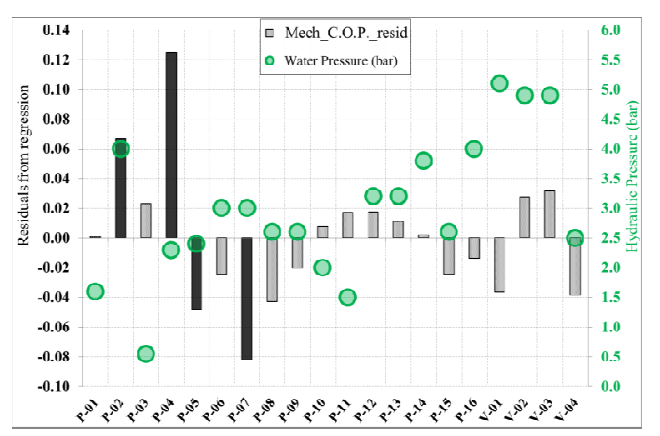

(b)

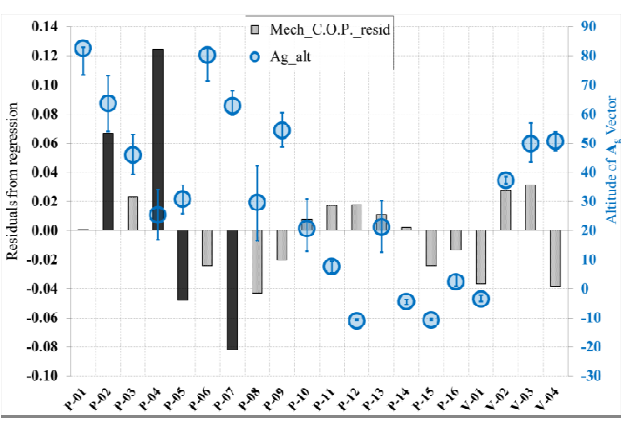

(c)

Fig. 6. Series of residuals from the computation of C.O.P.mech (observed minus computed values), along with water temperature (a), pressure (b), and altitude of vector $A_{g}(c)$. The biggest deviations are represented in bold.

An experiment carried out in April, 2014 with the same heat installation but without outward connections (adiabatic trap) showed a C.O.P around 1.7 (not shown). This result points to practical use of these installations.

Further research is needed, involving extensive and targeted experiment all around the year as well as at any time of the day in order to confirm the possible roles of the altitude of the vector $\mathrm{A}_{\mathrm{g}}$ and the seasonal changes of the IO extinction rate.

\section{Conclusions}

In the opinion of the authors, the results of the described experimental runs contribute to dissipate any doubt about the existence in nature and the detectability of a previously unknown interaction that, in contrast to the well-known ones, is not gauge invariant: potentials of physical fields are instrumental to its implementation, such potentials being 
considered as non-observable quantities in the standard physics. The results of the described experiments show a simple practical solution to the JTF problem using potentials of physical fields and byuons containing such potentials.

Moreover, from a practical point of view, the results demonstrate that the proposed new force of nature can be harnessed to substantially enhance the energy performance of simple, scalable and industrial-scale heat generators.

\section{Acknowledgments}

The authors gratefully acknowledge G.M. Lanini and G. Tagliaferri for assistance with the experimental stands and measurements; A. Crisci and F. Zabini for fruitful discussion; M. Pagliaro for support under the conferences SuNEC 2012 and SuNEC 2013. This research was partially funded by the Tuscany regional Government under the projects SUBCAL (Decree $\mathrm{N}^{\circ} .2460$, May 30 ${ }^{\text {th }}, 2012$ ) and T.I.L.A. (Decree $\mathrm{N}^{\circ}$. 6107 , December $\left.13^{\text {th }}, 2013\right)$.

\section{Appendix}

\section{Abbreviations Used in this Article}

$\begin{array}{ll}\mathrm{A} \Sigma & \text { Summary vector potential }(\mathrm{T} \cdot \mathrm{m}) \\ \mathrm{Ag} & \text { Cosmological vector potential }(\mathrm{T} \cdot \mathrm{m}) \\ \mathrm{BT} & \text { Byuon Theory } \\ \text { C.O.P. } & \text { Coefficient of Performance } \\ \text { C.O.P.mech } & \text { Mechanical Coefficient of Performance } \\ \text { IMF } & \text { Interplanetary Magnetic Field } \\ \text { IO } & \text { Information Object in the physical space } \\ \text { JTF } & \text { Joint Theory of Fields } \\ \text { SD } & \text { Special Device }\end{array}$

\section{References}

[1] Y.A. Baurov, E.Y. Klimenko, S.I. Novikov, Experimental observations of magnetic anisotropy, Dokl. Akad. Nauk SSSR. 315 (1990) 1116-1120.

[2] Y.A. Baurov, E.Y. Klimenko, S.I. Novikov, Experimental observation of space magnetic anisotropy, Phys. Lett. A. 162 (1992) 32-34.

[3] Y.A. Baurov, Space magnetic anisotropy and a new interaction in nature, Phys. Lett. A. 181 (1993) 283-288.

[4] Y.A. Baurov, A. V Kopaev, Experimental investigations of signals of a new nature with the aid of two high precision stationary quartz gravimeters, Hadron. J. 25 (2002) 697-712.

[5] Y.A. Baurov, On the structure of physical vacuum and a new interaction in Nature (Theory, Experiment and Applications), Nova Science, NY, 2000.

[6] Y.A. Baurov, Global Anisotropy of Physical Space, Experimental and Theoretical Basis, Nova Science, NY, 2004.

[7] Y.A. Baurov, V.L. Shutov, On the influence of vectorial magnetic potential of the Earth and the Sun on the $\beta$ - decay rate, Prikl. Fiz. 1 (1995) 40-45.
[8] Y.A. Baurov, Y.G. Sobolev, Y. V. Ryabov, V.F. Kushniruk, Experimental investigations of changes in the rate of beta decay of radioactive elements, Phys. At. Nucl. 70 (2007) $1825-1835$.

[9] Y.A. Baurov, A.A. Konradov, V.F. Kushniruk, E.A. Kuznetsov, Y.G. Sobolev, Y. V. Ryabov, et al., Experimental investigations of changes in beta-decay rate of $60 \mathrm{Co}$ and $137 \mathrm{Cs}$, Mod. Phys. Lett. A. 16 (2001) 2089-2101.

[10] Y.A. Baurov, V.A. Nikitin, V.B. Dunin, N.A. Demchuk, A.Y. Baurov, V. V Tihomirov, Results of experimental investigations of 60 Co beta-decay rate variation, Appl. Phys. 5 (2011) 12-21.

[11] Y.A. Baurov, A.A. Shpitalnaya, I.F. Malov, Global anisotropy of physical space and velocities of pulsars, Intl. J. Pure Appl. Phys. 1 (2005) 71-82.

[12] I.F. Malov, Y.A. Baurov, The distribution of space velocities of radio pulsars, Astron. Reports. 51 (2007) 830-835.

[13] Y.A. Baurov, I.F. Malov, On the Nature of Dark Matter and Dark Energy, J. Mod. Phys. 01 (2010) 17-32.

[14] Y.A. Baurov, I.B. Timofeev, V.A. Chernikov, S.F. Chalkin, A.A. Konradov, Experimental investigations of the distribution of pulsed-plasma-generator radiation at its various spatial orientation and global anisotropy of space, Phys. Lett. A. 311 (2003) 512-523.

[15] Y.A. Baurov, A.G. Znak, V.G. Farafonov, Experimental Investigation of Heat Content in the Jet of Magnetoplasmadynamic Accelerator in Accordance with its Spatial Orientation, in: Adv. Plasma Phys. Res. v. 5, Nova Science Publishers Inc, New York, 2007: pp. 179-196.

[16] Y.A. Baurov, A.A. Spitalnaya, A.A. Abramayan, V.A. Solodovnikov, Seismic activity of the earth, the cosmological vectorial potential and method of a short-term earthquakes forecasting, Nat. Sci. 03 (2011) 109-119.

[17] Y.A. Baurov, F. Meneguzzo, A.Y. Baurov, A.Y.J. Baurov, Plasma Vacuum Bubbles and a New Force of Nature, The Experiments, Int. J. Pure Appl. Sci. Technol. 11 (2012) 34-44.

[18] M.A. Margulis, Sonoluminescence, Physics-Uspekhi. 43 (2000) 259-282.

[19] Y.A. Baurov, Research of global anisotropy of physical space based on investigation of changes in $\beta$ and $\alpha$-decay rate of radioactive elements, motion of pulsars and anisotropy of cosmic rays, Am. J. Mod. Phys. 2 (2013) 177-184.

[20] Y.A. Baurov, I.F. Malov, F. Meneguzzo, Tornadoes and the global anisotropy of the physical space, Am. J. Mod. Phys. 3 (2014) 93-112.

[21] Y.A. Baurov, I.F. Malov, Variations of Decay Rates of Radioactive Elements and their Connections with Global Anisotropy of Physical Space, Int. J. Pure Appl. Phys. 6 (2010) 469-482. Also at: http://arxiv.org/abs/1001.5383.

[22] Y.A. Baurov, L. Albanese, F. Meneguzzo, V.A. Menshikov, Universal propulsion harnessing the global anisotropy of the physical space, Am. J. Mod. Phys. 2 (2013) 383-391.

[23] Y.A. Baurov, F. Meneguzzo, L. Albanese, Experimental investigation of the traction force for a new space thruster, Am. J. Astron. Astrophys. (2014) (in press). 